\title{
Adolescência e contracepção de emergência: Fórum 2005
}

\author{
Adolescence and emergency contraception: Forum 2005
}

Maria Ignez Saito ${ }^{1}$, Marta Miranda Lea/ ${ }^{2}$

\section{RESUMO}

Objetivo: Relatar os resultados do Fórum "Adolescência e Contracepção de Emergência”, que teve como proposta trazer maiores esclarecimentos sobre a contracepção de emergência (CE), além de apoio ético e técnico para sua prescrição, a partir da análise de três vertentes principais de discussão: o perfil da clientela adolescente; as questões éticas que envolvem a CE; a eficácia e o risco do método.

Fontes de dados: Discussão dos temas propostos a partir da literatura atual sobre o tema do Fórum organizado em 2005, que envolveu a participação de pediatras, ginecologistas, profissionais ligados a Comissões de Bioética, ao Conselho Federal de Medicina e ao Ministério da Saúde, advogados e juízes.

Síntese dos dados: A adolescência caracteriza-se por mudanças, transformações e experimentações que vinculam essa fase da vida à vulnerabilidade e risco. Alguns desses riscos estão relacionados ao exercício inadvertido ou impensado da sexualidade, cujas conseqüências são bem conhecidas: gravidez precoce, aborto e doenças sexualmente transmissíveis, entre as quais HPV e AIDS. É fundamental, portanto, que a assistência a essa faixa etária inclua o enfoque de prevenção, na qual a educação sexual seja vista como um processo do qual é parte relevante a orientação anticoncepcional, incluindo-se nesta a contracepção em situações de emergência.

Conclusões: $\mathrm{O}$ artigo apresenta as conclusões resultantes das discussões realizadas durante esse evento. Seu principal desdobramento foi a Resolução do Conselho Federal de Medicina: a CE não é abortiva e pode ser usada em qualquer etapa da vida reprodutiva.

Palavras-chaves: adolescente; bioética; gravidez na adolescência; anticoncepção pós-coito.

\section{ABSTRACT}

Objective: To report the results of the Forum "Adolescence and Emergency Contraception”. The objectives of this Forum were to enlighten and to bring ethical and technical support to the prescription of emergency contraception through the analysis of three main points: the profile of the adolescent clientele; the ethical issues involved in the emergency contraception; the risks and efficiency of this method.

Data sources: Discussion based on current literature on the theme in the Forum held in 2005, which included pediatricians, obstetricians, gynecologists, members of bioethics committees, members of the Federal Council of Medicine, delegates from the Ministry of Health, lawyers and judges.

Data synthesis: Adolescence includes change, transformation and experimenting, all of which lead to vulnerability and risk. Some of these are related to unoriented or unthought sexuality, whose consequences are well known: early pregnancy, abortion and sexually transmitted diseases, among which are HPV and HIV infections. Therefore, the medical assistance to adolescents must be focused on prevention, in which sexual education includes contraception orientation, particularly in emergency situations.

Conclusions: This review presents the conclusions that were achieved during the meeting. Its main consequence was a resolution by the Federal Council of Medicine stating that emergency contraception is not abortive and may be used at any stage of the reproductive life.

Key-words: adolescent; bioethics; pregnancy in adolescence; risk; contraception, postcoital.
1Professor livre docente pelo Departamento de Pediatria da Faculdade de Medicina da Universidade de São Paulo (FMUSP) e chefe da Unidade de Adolescentes do Instituto da Criança do Hospital das Clínicas da FMUSP ${ }^{2}$ Médica assistente da Unidade de Adolescentes do Instituto da Criança do Hospital das Clínicas da FMUSP e mestre em Medicina pelo Departamento de Pediatria da FMUSP
Endereço para correspondência:

Maria Ignez Saito

Alameda Ribeirão Preto, 546, apto. 7

CEP 01331-000 - São Paulo/SP

E.mail: mariais@icr.hcnet.usp.br

Recebido em: 6/3/2007

Aprovado em: 25/4/2007 


\section{Introdução}

Ao longo do século XIX, a adolescência passou a ser reconhecida como período crítico da existência humana ${ }^{(1-3)}$. Porém, esta fase da vida apresenta-se, freqüentemente, vinculada à vulnerabilidade e ao risco, inerentes às mudanças e transformações que a caracterizam ${ }^{(4)}$, o que torna fundamental o enfoque de prevenção.

Assim, durante a adolescência, devem ser considerados, entre outros, os riscos vinculados ao exercício inadvertido ou impensado da sexualidade, cujas consequiências são bem conhecidas: gravidez precoce, doenças sexualmente transmissíveis, entre as quais HPV e AIDS, aborto - conseqüências essas que podem levar à interrupção do projeto e/ou da qualidade de vida e até mesmo da própria vida ${ }^{(5)}$.

A gravidez na adolescência sempre constitui tema atual de discussão pelos problemas que acarreta, sendo importante considerar presente que as taxas de morbi-mortalidade são superiores para as mães adolescentes e seus conceptos e que os riscos são tanto maiores quanto mais baixa for a idade materna ${ }^{(6,7)}$.

O número de gravidezes neste período da vida, embora venha decrescendo nas faixas etárias mais avançadas, tem se mantido elevado mesmo em países desenvolvidos, sendo especialmente preocupante o aumento constatado nas idades mais baixas (dez a 14 anos) $)^{(7)}$.

Como agravos imediatos da gestação nessa faixa etária podem ser citados: anemia, eclâmpsia, desproporção céfalo-pélvica, hemorragia, parto prolongado, aborto e morte materna ${ }^{(8)}$. Uma palavra deve ser dita em relação ao risco a médio e longo prazo. Muitos autores apontam a dificuldade no estabelecimento das relações entre mãe e filho que extrapolam as condições socioeconômicas, pois a maternidade exige ajustes relevantes para que seja assumido o papel de mãe, o que é, muitas vezes, difícil para as adolescentes ${ }^{(9,10)}$.

Não se pode ainda deixar de mencionar que aproximadamente $25 \%$ das gestações não planejadas na adolescência evoluem para aborto, muitas vezes praticado com risco de mutilação ou, até mesmo, morte ${ }^{(11)}$.

Frente a uma problemática tão diversificada, a educação sexual surge como proposta fundamental de prevenção e deve ser vista como processo do qual é parte relevante a anticoncepção ${ }^{(12-14)}$, inclusive de emergência. Esta última ainda suscita dúvidas e reflexões, tendo, por esta razão, sido tema do Fórum 2005: "Adolescência e Contracepção de Emergência”, organizado pela Unidade de Adolescentes do Instituto da Criança do Hospital das Clínicas da Universidade de São Paulo (USP), coordenado pela professora doutora Maria Ignez
Saito e pela doutora Marta Miranda Leal, e realizado em 13 de agosto de 2005. Este evento contou com a participação de pediatras, ginecologistas, profissionais ligados a Comissões de Bioética, ao Conselho Federal de Medicina e ao Ministério da Saúde e foram escolhidos para respaldar os debates representantes da Justiça - advogados e juízes.

Utilizando-se de questões apresentadas como disparadores, esse grupo de trabalho procurou analisar a contracepção de emergência (CE) sob três vertentes fundamentais que levaram em conta: o perfil da clientela adolescente ${ }^{(15-21)}$; as questões éticas que envolvem a CE, inserida no contexto da contracepção na adolescência como um todo ${ }^{(22-29)}$; e a eficácia e o risco do método ${ }^{(30-52)}$.

O objetivo deste artigo é apresentar as conclusões resultantes das discussões realizadas durante esse evento e seus desdobramentos.

\section{Conclusões do Fórum}

A CE cumpre papel de destaque dentro da proposta de educação sexual, posto que seu caráter emergencial pode preceder o próprio processo educativo, sem deixar de fazer parte desse processo em suas etapas. Quando se leva em consideração as características e singularidades dos adolescentes, fica claro que a CE vai ao encontro de seu imediatismo, das constantes mudanças de pensar e sentir, colocando-se, portanto, como opção relevante de prevenção.

É reconhecido ser direito do adolescente ter acesso ao conhecimento de todos os métodos, inclusive da CE, identificando aqueles que ofereçam maior eficácia contraceptiva e proteção contra DST, levando-se em conta a autonomia de suas escolhas. E, é dever do profissional de saúde oferecer todas as alternativas de informação de forma clara, fácil, acessível, independente de qualquer circunstância. Partindo dessas premissas, é relevante enfatizar os conceitos de liberdade de escolha, de dupla proteção e incorporação da CE como parte de políticas públicas de saúde para os jovens.

Características dessa faixa etária poderiam conduzir ao uso mais freqüente, devendo redobrar-se a atenção dos profissionais no que diz respeito à queda de eficácia do método. A nova proposta da CE em dose única elimina a preocupação em relação ao uso incorreto pelas adolescentes, desde que não mais haverá a possibilidade do esquecimento da segunda dose.

$\mathrm{A}$ incorporação da $\mathrm{CE}$ às políticas públicas não só visa facilitar o acesso e treinar profissionais pra lidar com essa questão, mas também contribui para a formação de opinião. A realidade da iniciação precoce da atividade sexual e as 
estatísticas relevantes de gravidez e aborto na adolescência corroboram essa necessidade.

Colocada como estratégia de redução de danos, a CE deve participar efetivamente no projeto de educação sexual como um todo, para ambos os gêneros, quando se enfatiza um projeto de desenvolvimento da sexualidade de forma saudável, que torne o adolescente sujeito de suas escolhas.

Na realidade, a orientação da CE favorece a orientação do uso do condom, sendo que tanto outros métodos hormonais como a CE serão sempre complementares do preservativo. Lembrar que, além da falha mecânica, pode ocorrer a falha comportamental (por exemplo: o preservativo geralmente não é usado nas preliminares). Assim, até mesmo os trabalhos de prevenção da AIDS deveriam incluir informações sobre a CE.

De maneira mais ampla, considerou-se que a CE deva fazer parte de uma educação sexual que:

1. Considere seu caráter intersetorial (educação/saúde/cultura) e processual (processo contínuo, seqüencial, não pontual);

2. Inclua a educação de pares (multiplicadores adolescentes), aproveitando a tendência grupal do adolescente;

3. Tenha marcos de referência definidos (características da adolescência; conceito de gênero; conceito de saúde; direitos sexuais e reprodutivos; metodologias participativas);

4. Destaque o auto-cuidado e o cuidado do outro;

5. Ressalte o prazer;

6. Não seja meramente uma proposta sexual "medicalizada";

7. É importante reiterar que uma política de saúde sexual que promova ações coletivas deve começar pela sensibilização e treinamento para técnicos.

Em relação aos riscos de utilização da $\mathrm{CE}$, nenhum foi ainda definitivamente demonstrado. Deve ser esclarecido que alguns riscos temidos não foram demonstrados:

1. Ação como método abortivo teratogênico, em caso da adolescente já estar grávida no momento da tomada do contraceptivo de emergência;

2. Facilitação da aquisição de DST/AIDS;

3. Banalização do seu uso, passando a ser usado como método contraceptivo regular;

4. Passaporte para vulgarização da relação sexual na adolescência - promiscuidade. Não se deve culpar a CE pela promiscuidade; as origens ou causas da mesma estão na própria sociedade, na questão de gênero, no meio onde o adolescente se encontra inserido, na ausência de projeto de vida, auto-estima e auto-cuidado;

5. Alteração do eixo hipotalâmico-hipofisário-gonadal definitiva, mesmo frente ao uso repetitivo. Fazem-se necessários estudos multicêntricos com metodologia qualitativa e quantitativa para melhor entendimento do risco hipotético do uso habitual do método.

Algumas preocupações procedem e servem de alerta para que dúvidas possam ser sanadas, devendo-se destacar os riscos já comprovados:

1. O método é pouco eficaz se utilizado repetidamente (a curtos intervalos); na realidade, o único risco real do contraceptivo de emergência é de falha do método, pois é o menos eficaz entre os métodos modernos para contracepção rotineira;

2. A adolescente não deve se acreditar protegida até o próximo ciclo menstrual, sendo fundamental a orientação sobre uso de outro método ou abstinência até a próxima menstruação;

3. Na experiência da maioria dos profissionais, a CE é mais subutilizada do que superutilizada;

4. O uso inadequado, infelizmente, muitas vezes decorre de problemas mais relacionados ao despreparo dos profissionais de saúde, à venda equivocada nas farmácias e às estratégias de divulgação antiéticas;

5. Não houve consenso quanto à prescrição prévia do contraceptivo de emergência para adolescentes:

a) A maioria advoga a pertinência da mesma dentro de uma proposta de prevenção. Receitar é direito e dever do médico. Avaliar risco é papel do médico. Portanto, ele pode prescrever a CE para a adolescente nas consultas de prevenção e fornecer a receita para os casos que considerar de risco pessoal, biológico, social, familiar e psicológico;

b) Os profissionais que não concordaram com o fornecimento da receita prévia e assim se posicionaram por acreditarem que a CE não deve ser enfatizada numa consulta de prevenção, imaginando que isso possa intervir no comportamento adequado por parte do adolescente, quando da orientação da contracepção regular.

Discutindo-se estratégias para facilitar o acesso e o uso do contraceptivo de emergência, foi sugerido que deveria ser parte das ações de prevenção das Unidades Básicas de Saúde/Unidades de Saúde da Família (UBS/USF). Nas localidades que não contassem com UBS/USF abertas nos fins de semana, seria estabelecido o acesso a um Pronto Socorro (PS) de referência. Neste último caso, uma opção ao PS seria a dispensação, sendo entregue a algumas farmácias (de plantão), que forneceriam o contraceptivo de emergência ao usuário, retendo sua receita, por meio da qual seria ressarcido posteriormente. 
Independentemente da idade, os profissionais valorizaram a autonomia de decisão e capacidade do adolescente de administrar suas tarefas. Assim, cabe a orientação e o uso da CE em menores de 14 anos, não existindo infração ética nessa prescrição, respeitadas as ressalvas do artigo 103 do Código de Ética Médica. Quanto à implicação legal, novamente, deve ser salientado que a presunção de violência desaparece mediante as informações que o profissional possui da sua não existência, e que devem ser registradas em prontuário.

Em relação aos direitos sexuais e reprodutivos dos adolescentes, cabe lembrar as seguintes premissas:

1. A afirmação e construção do conceito de direitos reprodutivos vêm se dando, basicamente, no campo da saúde, o que implica, por vezes, restringi-lo às questões de saúde sexual e reprodutiva. Portanto, um grande desafio para a afirmação do novo conceito é não permitir sua restrição às questões de saúde, mas aportá-lo na esfera da cidadania plena, buscando tratá-lo na sua dimensão política, ou seja, "como prerrogativa de autonomia e liberdade dos sujeitos humanos nas esferas da sexualidade e reprodução.

2. O Estatuto da Criança e do Adolescente (ECA) - Lei $n^{\circ}$ 8.069 de 13/07/90 - claramente dispõe a prioridade do atendimento médico do adolescente, o direito à autonomia e sua absoluta proteção à vida e à saúde de forma que permita seu desenvolvimento sadio e harmonioso. Em nenhum momento, o ECA condicionou o acesso do adolescente a esses serviços ou direitos, por meio da anuência de seus pais ou responsáveis, por outro lado, garante que toda criança ou adolescente seja ouvida e sua opinião considerada no momento de decidir sobre fatos que envolvam sua vida íntima. Portanto, os direitos dos adolescentes à assistência sexual e reprodutiva consistem em direitos personalíssimos garantidos pelo ECA e podem ser exercidos independentemente da autorização da família ou responsável.

3. É importante incluir uma das principais conclusões do "Fórum 2002 - Contracepção, Adolescência e Ética”: "o respeito da autonomia da criança e do adolescente, o que implica para este último em privacidade e confidencialidade, faz com que esses indivíduos passem de objeto a sujeito de direito". O adolescente tem direito à educação sexual, ao acesso à informação sobre contracepção, à confidencialidade e ao sigilo sobre sua atividade sexual e sobre a prescrição de métodos anticoncepcionais, respeitadas as ressalvas acima (Art. 103, Código de Ética Médica). O profissional que assim se conduz não fere nenhum precei- to ético, não devendo temer nenhuma penalidade legal. "Porém, nem a Constituição Federal, nem o Estatuto da Criança e do Adolescente abordam expressamente os direitos sexuais e reprodutivos dos jovens, merecendo revisão legislativa ou interpretação satisfatória que atenda aos objetivos traçados nos documentos internacionais".

As orientações, quando da prescrição da CE dirigidas a ambos os gêneros, incluem:

1. Orientar quanto às indicações e quanto à dosagem (priorizar as apresentações contendo apenas levonorgestrel, pela sua maior eficácia, e a tomada em dose única, por aumentar a adesão);

2. Reforçar que a CE é o último passo antes da ocorrência ou da possibilidade de uma gravidez, portanto deve ser entendida como uma opção que só está sendo utilizada porque os métodos contraceptivos falharam ou porque não foram utilizados;

3. Orientar que não é eficaz como método de rotina, devendo ser utilizado em casos emergenciais; além disso, seu uso inadequado, repetitivo, aumenta a ineficácia do método;

4. Orientar sobre a ocorrência de efeitos colaterais leves, nos primeiros dois dias após tomada da medicação, como cefaléia, náuseas e vômitos;

5. Se vomitar nas primeiras duas horas após a ingestão do contraceptivo de emergência, orientar sobre a necessidade de repetir a mesma dosagem do medicamento;

6. Esclarecer que não interfere na evolução de gravidez já estabelecida e que não é teratogênico;

7. É um método seguro, sem condições médicas que contraindiquem seu uso; pode ser utilizado por pacientes com doenças crônicas;

8. Ideal usar o mais próximo possível da relação sexual desprotegida; a eficácia é maior quanto mais precoce for a tomada, mas pode ser utilizado até cinco dias após ato sexual desprotegido;

9. A menstruação é esperada para mesma data ou com pequenas variações, antecipando ou atrasando o fluxo por cerca de sete dias;

10.Não ter relações sexuais ou usar outro método contraceptivo (método de barreira, por exemplo) até a próxima menstruação, pois a CE confere proteção somente para aquele evento, não garantindo prevenção seqüencial;

11. Esclarecer sobre mecanismo de ação, que não é abortivo por não atuar após a nidação;

12.Se não menstruar em três semanas, pensar na possibilidade de gravidez; 
13.Identificar a adolescente que faz uso do contraceptivo de emergência como adolescente de risco e aproveitar o momento da prescrição do contraceptivo de emergência para iniciar a orientação sexual de forma ampla, reforçando a necessidade de adesão à dupla proteção;

14.Lembrar que, nesse momento, a adolescente poderá não estar disponível emocionalmente para apreender todos os conhecimentos necessários e tomar decisões, havendo necessidade, portanto, de retornos posteriores e inclusão da paciente no programa de atenção à saúde do adolescente da Unidade de Saúde;

15.É importante ter presente que outro grupo que se beneficia com esse método, nas situações de emergência, é o dos portadores de doenças crônicas, mesmo os que são impossibilitados de usarem anticoncepcionais hormonais de rotina que contenham estrógeno;

16. Os participantes do Fórum acreditam que se deva abolir o termo da "pílula do dia seguinte", pois isto pode confundir a adolescente quanto ao uso correto, ao sugerir que só possa ser utilizado nas primeiras 24 horas, ou confundir o profissional ao identificá-la como a RU486, droga abortiva que nunca foi aprovada para uso no Brasil.

\section{Sugestões}

Algumas sugestões elaboradas neste segundo Fórum seguem-se abaixo:

1. Parceria da Saúde com os meios de comunicação (mídia) para o exercício de uma sexualidade com responsabilidade, abrangendo propostas educativas e preventivas;

2. Execução de trabalhos multicêntricos nacionais para reconhecimento do comportamento dos adolescentes frente à CE, com avaliação do uso e dos desdobramentos da CE. Para isso é necessária a elaboração de protocolos de pesquisa e o emprego das técnicas de medicina baseada em evidência;

3. Proposta junto à rede pública - acesso mais rápido aos atendimentos nas Unidades de Saúde; equipe de acolhimento preparada para orientação e informação. Trabalho de sensibilidade e educativo junto aos profissionais de PS e Pronto Atendimento com kit e protocolo para CE.

4. Proposta de elaboração de uma resolução no Conselho Federal de Medicina (CFM) sobre CE para orientar e respaldar o profissional. A exposição de motivos dessa resolução deve se basear nas conclusões deste fórum.

\section{Desdobramentos}

A CE já faz parte do Marco Teórico Referencial da Saúde Sexual e Reprodutiva do Adolescente e do Marco Legal "Saúde, um direito de adolescentes”, ambos do Ministério da Saúde. Além disso, foi aprovada pelo CFM, após discussões com representantes do Fórum, uma resolução publicada em diário oficial em janeiro de 2007 (Seção 1, Edição no. 12, 17/1/2007) que estabeleceu como definitiva a condição não abortiva da $\mathrm{CE}$, validando seu uso em qualquer etapa da vida reprodutiva. O conteúdo desta resolução é aqui apresentado no Anexo.

\section{Considerações finais}

A CE cumpre papel de destaque dentro da proposta de educação sexual, devendo fazer parte da orientação anticoncepcional como um todo, posto que é um direito dos adolescentes terem acesso ao conhecimento sobre todos os métodos contraceptivos.

Com a antecipação da puberdade e do início da atividade sexual, cada vez mais a anticoncepção faz parte da proposta pediátrica de prevenção de problemas ou agravos à saúde, ainda que possam persistir alguns aspectos polêmicos para sua prescrição. Este Fórum teve como principal contribuição esclarecer alguns desses aspectos. Pôde-se concluir que a CE não é um método abortivo, não leva reconhecidamente ao abandono de outros métodos ou incremento da promiscuidade e pode ser usada em qualquer etapa da vida reprodutiva.

\section{Agradecimentos}

Participantes do Forum 2005 “Adolescência e Contracepção de Emergência”: Albertina Duarte Takiuti, Alexandre Maschio, Anísio de Moura, Benito Lourenço, Clóvis Constantino, Cristina Guazzelle, Déa Mascarenhas Cardozo, Débora Gejer, Eleuse Brito Guimarães, Fermino Magnani, Fernando Manuel M. Cruz, Geni Beznos, Herlander Manoel M. Coelho, Isabel Carmen, João Luiz P. Silva, João Tadeu L. dos Reis, Jorge Andalaft, José Ricardo Ayres, Júlia Valéria Cordeline, Lígia de Fátima N. Reato, Lucia Helena S. C. Paiva, Lucimar Aparecida Françoso, Magda Motta, Marco Aurélio Galetta, Maria Helena Rusany, Maria Ignez Saito, Maria Ivone F. Oliveira, Maria José C. Sant'Anna, Maria Luisa Zenaide, Maria Regina D. Azevedo, Maria Stela M. Santana, Maria Verônica G. Coates, Mariângela Barbosa, Marisa Andalaft, Marta Miranda Leal, Maurício Castro S. Lima, Míriam Ventura da Silva, Paulo Ramires Sant’Anna, Pilar Gutierrez, Regina Figueiredo, Sandra Martins, Tamara Goldberg, Teresa de Lamare Franco Netto, Waldi Craveiro Bezerra, Williams Santos Ramos. 
Edição Número 12 de 17/01/2007

\section{Conselho Federal de Medicina}

\section{Entidades de Fiscalização do Exercício das Profissões Liberais}

\section{RESOLUÇÃO N. ${ }^{\circ} 1.811$, DE 14 DE DEZEMBRO DE 2006}

Estabelece normas éticas para a utilização, pelos médicos, da Anticoncepção de Emergência, devido a mesma não ferir os dispositivos legais vigentes no país.

O Conselho Federal de Medicina, no uso das atribuições conferidas pela Lei nº 3.268, de 30 de setembro de 1957, alterada pela Lei $\mathrm{n}^{\circ} .11 .000$, de 15 de dezembro de 2004, regulamentada pelo Decreto no. 44.045, de 19 de julho de 1958, e CONSIDERANDO que o direito reprodutivo funda-se nos princípios da dignidade da pessoa humana e propicia o exercício da paternidade responsável;

CONSIDERANDO que compete ao Estado propiciar recursos educacionais, científicos e materiais para o exercício desse direito, sendo vedada qualquer ação coercitiva por parte de entidades públicas ou privadas;

CONSIDERANDO que no Brasil há um número significante de mulheres expostas à gravidez indesejada, seja pelo não uso ou uso inadequado de métodos anticoncepcionais;

CONSIDERANDO que as faixas mais atingidas são as de adolescentes e de adultas jovens, que, freqüentemente, iniciam a atividade sexual antes da anticoncepção;

CONSIDERANDO que a prevenção da gravidez indesejada constitui bom exemplo de sexualidade responsável, e que tal gravidez pode conduzir a custos psíquicos e sociais por vezes irreversíveis;

CONSIDERANDO que a prática da dupla proteção - recomendada pela Organização Mundial da Saúde, Ministério da Saúde, Federação Brasileira das Sociedades de Ginecologia e Obstetrícia e Sociedade Brasileira de Pediatria -, busca incutir a utilização da camisinha masculina ou feminina, concomitante a um outro método anticoncepcional, incluindo-se a Anticoncepção de Emergência;

CONSIDERANDO que a Anticoncepção de Emergência pode ser utilizada em qualquer etapa da vida reprodutiva e fase do ciclo menstrual na prevenção da gravidez e que, em caso de ocorrência de fecundação, não haverá interrupção do processo gestacional; CONSIDERANDO que o objetivo da Anticoncepção de Emergência é evitar a gravidez e que mesmo nos raros casos de falha do método não provoca danos à evolução da gestação;

CONSIDERANDO que a Anticoncepção de Emergência poderá contribuir para a diminuição da gravidez indesejada e do aborto provocado;

CONSIDERANDO, finalmente, o decidido na sessão plenária realizada em 14 de dezembro de 2006, resolve:

Art. $1^{\circ}$ Aceitar a Anticoncepção de Emergência como método alternativo para a prevenção da gravidez, por não provocar danos nem interrupção da mesma.

Art. $2^{\circ}$ Cabe ao médico a responsabilidade pela prescrição da Anticoncepção de Emergência como medida de prevenção, visando interferir no impacto negativo da gravidez não planejada e suas consequiências na Saúde Pública, particularmente na saúde reprodutiva.

Art. $3^{\circ}$ Para a prática da Anticoncepção de Emergência poderão ser utilizados os métodos atualmente em uso ou que porventura sejam desenvolvidos, aceitos pela comunidade científica e que obedeçam à legislação brasileira, ou seja, que não sejam abortivos. Art. $4^{\circ}$ A Anticoncepção de Emergência pode ser utilizada em todas as etapas da vida reprodutiva.

Art. $5^{\circ}$ Revogam-se todas as disposições em contrário.

Art. $6^{\circ}$ Esta resolução entra em vigor a partir da data de sua publicação.

EDSON DE OLIVEIRA ANDRADE

Presidente do Conselho

LÍVIA BARROS GARÇÃO

Secretária-Geral 


\section{Referências bibliográficas}

1. Violato C. History of adolescence. In: Comprehensive adolescent health care. $1^{\text {st }}$ ed. USA: Stanford Friedman; 1992. p.3.

2. Grossman E. O médico de adolescentes e o seu ofício: reflexões sobre as normas e a prática [tese de mestrado]. Rio de Janeiro (RJ): Instituto Fernandes Figueira da Fundação Oswaldo Cruz; 1995.

3. Muss R. Theories of adolescence. $4^{\text {th }}$ ed. New York: Random House; 1982.

4. Leal MM, Saito MI. Síndrome da adolescência normal. In: Saito MI, Silva LEV, editores. Adolescência: prevenção e risco. $1^{a}$ ed. São Paulo: Atheneu; 2001. p. 105-14.

5. Saito MI. Sex education in school: preventing unwanted pregnancy in adolescents. Int J Gynecol Obstet 1998;63:1S57-60.

6. Guimarães EMB. Gravidez na adolescência: fatores de risco. In: Saito MI, Silva LEV, editores. Adolescência: prevenção e risco. $1^{\mathrm{a}}$ ed. São Paulo: Atheneu; 2001. p. 291-8.

7. Pinto e Silva JL, Motta ML. Gravidez na adolescência. In: Neme B. Obstetrícia básica. $1^{a}$ ed. São Paulo: Sarvier; 1994. p. 886-9.

8. Galletta MA, Lippi AT, Giribola A, Miguelez J, Zugaib M. Resultados obstétricos e perinatais em gestantes adolescentes atendidas em pré-natal especializado. Rev Ginecol Obstet 1997;8:10-9.

9. Furstenberg FF. Burdens and benefits: the impact of early childbearing on the family. J Soc Issues 1980;36:64-87.

10. Lizarraga JL, Maehr JC, Wingard DL, Felice ME. Psychosocial and economic factors associated with infant feeding intentions of adolescent mothers. J Adolesc Health 1992;13:676-81.

11. Olukoya AA, Kaya A, Ferguson BJ, AbouZahr C. Unsafe abortion in adolescents. Int J Gynaecol Obstet 2001;75:137-47.

12. Saito MI. Sexualidade, adolescência e orientação sexual: reflexões e desafios. Rev Med (São Paulo) 1996;75:26-30.

13. Saito MI, Leal MM. O exercício da sexualidade na adolescência: a contracepção em questão. Pediatria (São Paulo) 2003;25:36-42.

14. World Health Organization. Department of Reproductive Health Research. Selected practice recommendations for contraceptive use. $2^{\text {nd }}$ ed. Geneva: WHO; 2004.

15. Rowlands S, Devalia H, Lawrenson R, Logie J, Ineichen B. Repeated use of hormonal emergency contraception by younger women in the UK. Br J Fam Plann 2000;26:138-43.

16. Free C, Lee RM, Ogden J. Young women's accounts of factors influencing their use and non-use of emergency contraception: in-depth interview study. BMJ 2002;325:1393-7.

17. Pollack JS, Daley AM. Improve adolescents' access to emergency contraception. Nurse Pract 2003;28:11-23.

18. Irwin CE. Emergency contraception for adolescents: the time to act is now. J Adolesc Health 2004;35:257-8.

19. Trussell J. Contraceptive failure in the United States. Contraception 2004;70:89-96.

20. Walker DM, Torres P, Gutierrez JP, Flemming K, Bertozzi S. Emergency contraception use is correlated with increased condom use among adolescents: results from Mexico. J Adolesc Health 2004;35:329-34.

21. Litt IF. Placing emergency contraception in the hands of women. JAMA 2005;293:98-9

22. Estatuto da Criança e do Adolescente (ECA). Lei n. 8.069, de 13 de julho de 1990. [citado em 18 de abril de 2007]. Disponível em: www.mj.gov.br/sedh/ct/conanda/eca3.pdf

23. Ministério da Saúde. Prevenção e tratamento dos agravos resultantes da violência sexual contra mulheres e adolescentes. Norma técnica 1ª edição. Brasilia, 1998. Disponivel em: www.saude.gov.br/programas /mulher/norviol.html

24. Committee on Adolescence. American Academy of Pediatrics. Emergency contraception. Pediatrics 2005;116:1026-35.

25. Gold MA, Sucato GS, Conard LA, Hillard PJ. Provision of emergency contraception for adolescents. J Adolesc Health 2004;35:66-70.

26. Sociedade Brasileira de Pediatria, Federação Brasileira das Sociedades de Ginecologia e Obstetrícia. Adolescência, anticoncepção e ética: diretrizes [citado em 18 de abril de 2007]. Disponivel em: http://www.febrasgo.org.br/adolescencia.htm

27. Society for Adolescent Medicine. Confidential health care for adolescents: position paper of the Society for Adolescent Medicine. J Adolesc Health 2004;35:160-7.

28. Society for Adolescent Medicine. Provision of emergency contraception to adolescents: Position paper of the Society for Adolescent Medicine. J Adolesc Health 2004;35:66-70.

29. Ventura M. Direitos reprodutivos no Brasil. São Paulo: Câmara Brasileira do Livro, 2004.
30. Glasier A, Baird D. The effects of self-administering emergency contraception. N Engl J Med 1998;339:1-4.

31. Task force for postovulatory methods of fertility regulation. Randomised controlled trial of levonorgestrel versus Yuzpe regimen of combined oral contraception for emergency contraception. Lancet 1998;352:428-33.

32. Raine T, Harper C, Leon K, Darney P. Emergency contraception: advance provision in a young, high-risk clinic population. Obstet Gynecol 2000;96:1-7.

33. Ellertson C, Ambardekar S, Hedley A, Coyaji K, Trussell J, Blanchard K. Emergency contraception: randomized comparison of advance provision and information only. Obstet Gynecol 2001;98:570-5.

34. Ellertson C, Trussell J, Stewart F, Koenig J, Raymond EG, Shochet T. Emergency contraception. Semin Reprod Med 2001;19:323-30

35. Rodrigues I, Grou F, Joly J. Effectiveness of emergency contraceptive pills between 72 and 120 hours after unprotected sexual intercourse. Am J Obstet Gynecol 2001;184:531-6.

36. Turner AN, Ellertson C. How safe is emergency contraception? Drug Safety 2002;25:695-706.

37. WHO Research group on post-ovulatory methods of fertility regulation. Low dose mifepristone and two regimens of levonorgestrel for emergency contraception: a WHO multicentre randomized trial. Lancet 2002;360:1803-10.

38. Belzer M, Yoshida E, Talar T, Tucker D, Chung K, Sanchez K. Advanced supply of emergency contraception for adolescent mothers increased utilization without reducing condom or primary contraception use. J Adolesc Health 2003:32:122-3.

39. Croxatto HB, Ortiz ME, Müller AL. Mechanisms of action of emergency contraception. Steroids 2003;68:1095-8.

40. Ellertson C, Evans M, Ferden S, Leadbetter C, Spears A, Johnstone K et al. Extending the time limit for starting the Yuzpe regimen of emergency contraception to 120 hours. Obstet Gynecol 2003;101:1168-71.

41. Gainer E, Blum J, Toverud E-L, Portugal N, Tyden T, Nesheim B-l et al. Bringing emergency contraception over the counter: experiences of nonprescription users in France, Norway, Sweden and Portugal. Contraception 2003;68:117-24.

42. International consortium for emergency contraception. Emergency contraceptive pills: medical and service delivery guidelines. $2^{\text {nd }}$ ed. Washington: The International Consortium for Emergency Contraception; 2003.

43. Jackson RA, Schwarz EB, Freedman L, Darney P. Advance supply of emergency contraception: effect on use and usual contraception - a randomized trial. Obstet Gynecol 2003;102:8-16.

44. Westhoff C. Clinical practice. Emergency contraception. N Engl J Med 2003;349:1830-5.

45. Checa MA, Pascual J, Robles A, Carreras S. Trends in the use of emergency contraception. An epidemiological study in Barcelona, Spain (1994-2002). Contraception 2004;70:199-201.

46. Figueiredo R. Contracepção de emergência no Brasil: necessidade, acesso e política nacional [citado em 18 de abril de 2007]. Disponível em www.ipas.org. $\mathrm{br} /$ revista/set04.html

47. Gold MA, Wolford JE, Smith KA, Parker AM. The effects of advance provision of emergency contraception on adolescent women's sexual and contraceptive behaviors. J Pediatr Adolesc Gynecol 2004;17:87-96.

48. Lo SS, Fan SY, Ho PC, Glasier AF. Effect of advanced provision of emergency contraception on women's contraceptive behavior: a randomized controlled trial. Hum Reprod 2004;19:2404-10.

49. Bissell P, Anderson C. Enhanced access to emergency contraception. Lancet 2005;365:1668-70.

50. Ministério da Saúde. Anticoncepção de emergência: perguntas e respostas para profissionais de saúde. Brasília, 2005. (Série Direitos Sexuais e Direitos Reprodutivos, caderno n.3) [citado em 18 de abril de 2007]. Disponível em: www.redece.org/manualce2005.pdf

51. Raine T, Harper CC, Rocca CH, Fischer R, Padian N, Klausner JD et al. Direct access to emergency contraception through pharmacies and effect on unintended pregnancy and STIs - a randomized controlled trial. JAMA 2005;293:54-62.

52. Ziebland S, Wyke S, Seaman P, Fairhurst K, Walker J, Glasier A. What happened when Scottish women were given advance supplies of emergency contraception? A survey and qualitative study of women's views and experiences. Soc Sci Med 2005;60:1767-79. 